\title{
JUVENTUD Y CONSUMO: BASES ANALÍTICAS PARA UNA PROBLEMATIZACIÓN
}

OMAR CATALÁN MARÍN*

\begin{abstract}
RESUMEN
El presente ensayo presenta un marco conceptual de base que permita abordar los procesos de consumo en los jóvenes, desde una perspectiva relacional. En primera instancia, se muestra un contexto macrosocial que define a las sociedades que poseen una cultura de consumo y su relación con la juventud. Con posteridad, se desarrolla un conjunto de elementos psicosociales y culturales del consumo para un análisis ulterior. Finalmente se hace una presentación relacional de los elementos conceptuales que permitan orientar la interpretación del consumo en la juventud, dentro de un contexto social específico; esto es, en las sociedades de consumo.
\end{abstract}

\section{PALABRAS CLAVE: IDENTIDAD, PERSONALIZACIÓN, VALOR DE CAMBIO SEMÁNTICO}

* Licenciado en Sociología, Universidad de Playa Ancha, Valparaíso, Chile. Correo electrónico: omarcatalanmar@gmail.com. 


\title{
JUVENTUDE E CONSUMO: BASES ANALÍTICAS PARA UMA PROBLEMATIZAÇÃO
}

\begin{abstract}
RESUMO
O presente ensaio apresenta um marco conceitual de base que permita abordar os processos de consumo dos jovens a partir de uma perspectiva relacional. Primeiramente, apresenta-se um contexto macrossocial que define as sociedades que possuem uma cultura de consumo e sua relação com a juventude. Em seguida, desenvolve-se um conjunto de elementos psicossociais e culturais do consumo para uma análise futura. Por último, faz-se uma apresentação relacional dos elementos conceituais que permitem orientar a interpretação do consumo da juventude dentro de um contexto social específico, isto é, dentro das sociedades de consumo.
\end{abstract}

PALAVRAS-CHAVE: IDENTIDADE, PERSONALIZAÇÃO,
VALOR DE MUDANÇA SEMÂNTICA

YOUTH AND CONSUMPTION: ANALYTICAL FRAMEWORKS FOR PROBLEMATIZATION

\begin{abstract}
This presents a basic conceptual framework that addresses the processes of consumption among youth from a relational perspective. It comes in the first instance as a macro-social context that defines societies with a consumer culture and its relationship with youth. Subsequently it develops a set of psychosocial and cultural elements of consumption for further analysis. Eventually it develops a relational presentation of the conceptual elements to guide the interpretation of consumption in youth within a specific social context, that is, within consumer societies.
\end{abstract}

KEY WORDS: IDENTITY, PERSONALIZATION, VALUE OF SEMANATIC CHANGE 


\section{SOCIEDAD DE CONSUMO Y JUVENTUD. CONSIDERACIONES INICIALES}

DE MANERA PRELIMINAR, MOSTRAREMOS una visión general que defina a las sociedades de consumo y su relación cultural con la juventud, para así establecer las bases contextuales desde donde expondremos. Posteriormente, desarrollaremos un cuerpo de elementos conceptuales que orienten un análisis, comprensión y problematización inicial del consumo en los jóvenes y su relación con el mercado, considerando a este último como un espacio de vital importancia en la construcción y afirmación de las identidades y en la integración social en sociedades que se rigen por una lógica de mercado.

En primer lugar, debemos considerar qué entendemos cuando hablamos de consumo y de una sociedad de consumo.

El objeto que se consume - sea un bien o un servicio-, en una dimensión básica, satisface una necesidad específica. No obstante, si sólo consideramos la función de satisfacer, la acción de consumir remitiría hacia una actividad esencialmente pasiva, en circunstancias de que el consumo es, principalmente, un proceso, una dinámica de relación sistemática dentro de un sistema cultural. En la acción de consumir, se establece una interacción constante entre signos y connotaciones sociales atribuidas a las mercancías y a su relación con los consumidores. Por ello, podemos considerar que en el consumo hay una componente social que se manifiesta en un espacio determinado - el mercado- - el que fija un sistema de conductas, actitudes y relaciones humanas producto de aquello.

Dentro de un marco sociocultural, se puede considerar que el consumo constituye una organización del ambiente como sustancia significante que dota al espacio (social y físico) de significados determinados, proyectados y absorbidos en las relaciones sociales. Es, en definitiva, una actividad de manipulación de signos (Baudrillard, 1969).

De acuerdo a esto, la acción de consumo no recae necesariamente en las personas, sino en el producto del conjunto de relaciones concretas y simbólicas que mantienen entre ellas, y que se vinculan entre sí. Por consiguiente, el objeto de consumo se concibe como aquella red de pautas culturales, connotaciones y signos en donde los bienes se organizan y adquieren significado (Marinas, 2000). Según esto, la vida social mediada por el mercado, en un grado específico, se estructura en relación con el conjunto de significados que los bienes expresan y que son asimilados por las personas mediante la adquisición de mer- 
cancías. Así, frente a otros, proyectan una identidad específica que les permite llevar a cabo sus proyectos de vida, según parámetros socialmente valorados.

Por otro lado, al hablar de sociedades de consumo hacemos referencia a que en ellas el consumo se constituye en un acuerdo social, fuerza de impulso y desarrollo de una comunidad que lo adopta como una cultura específica e interpela a sus ciudadanos en función del modelo cultural. De este modo, el consumo coordina y ocupa un rol importante en la reproducción sistémica, en la integración y la estratificación social, en la formación y construcción de las identidades y, asimismo, en la selección y consecución de prácticas socialmente relevantes. Igualmente, el consumo se presenta como factor principal de estratificación, inclusión y exclusión social. Por lo tanto, podemos mencionar que en las sociedades de consumo, mediadas por el mercado de manera estructural, se reinterpretan las relaciones humanas a semejanza de la dinámica que se establece entre consumidores y bienes de consumo (Bauman, 2005; 2007). Así, el mercado se establece como un espacio socialmente institucionalizado.

Dentro de este espacio social, las relaciones humanas se convierten en relaciones de consumo: la interacción se consuma a través de las mercancías, las cuales se transforman en mediaciones necesarias para la comunicación entre los sujetos (Baudrillard, 1969). Consecuentemente, la interacción misma se convierte en objeto de consumo: se abstrae mediante bienes con significados específicos que las personas integran.

En otro extremo, a la sociedad de consumo se la considera auge y expansión de valores hedonistas, donde el sujeto, libre de las trabas coercitivas y disciplinarias de una sociedad conservadora, se transforma en el principal articulador de sus proyectos de vida. De esa manera, se crea una cultura hedonista que permite a las personas dedicarse a vivir más para sí; serán ellas quienes autodeterminan su relación con otros y consigo mismas (Lipovetsky, 1990; 2002). Con ello, la dinámica del consumo en las sociedades contemporáneas permitiría un desarrollo de valores democráticos por el hecho de permitir el selfservice —autorrealización-, que otorga más libertades a los sujetos. Según esta lógica, desaparecen paulatinamente los modelos de sociedades rigurosas y panópticas, que tienden al control social.

Desde la segunda mitad del siglo $\mathrm{XX}$, al término de la segunda guerra mundial y posteriormente con la crisis del Estado de Bienestar, se comenzó a consolidar una lógica de mercado que penetraba en casi todos los ámbitos de la vida pública y privada. En esta etapa de la 
sociedad moderna - en el hemisferio occidental y en los países sin regímenes comunistas - la lógica del libre mercado comenzó a ganar terreno y trajo consigo nuevos medios de interacción social. Entre otros: desarrollo de la publicidad, avance tecnológico, consumo de masas (Lipovetsky, 1990). Se manifestó así un cambio constante en las relaciones tradicionales que las personas mantenían con el mercado: cada vez más, el mercado fue soberano en el ámbito social, lo que provocó serios cambios en la cultura y permitió la consolidación de sociedades con un capitalismo de consumo.

Los cambios culturales se presentaron de preferencia en las grandes metrópolis de aquel entonces, especialmente en Europa occidental y Estados Unidos, y conforme finalizaban las últimas décadas del siglo XX se manifestaron en los países subdesarrollados.

En las principales ciudades del hemisferio norte occidental comenzaron a emerger gran cantidad de subculturas juveniles de expresión espectacular. Y fue la primera generación de posguerra la que experimentó los incipientes cambios en la relación con el mercado y las formas que adoptaba el consumo.

Según lo precedente, se puede considerar que desde el período de posguerra los jóvenes experimentaron una socialización más por el ambiente externo que por el tradicional familiar, mediante nuevas formas de interacción social. La publicidad, la moda, la constante extensión de la educación, los medios de comunicación de masas, el aumento de capital adquisitivo y toda una industria de producción dedicada a ellos, entre otros elementos, adquirieron especial importancia en sus concepciones y proyectos de vida. En los jóvenes existían prácticas de consumo específicas que incidieron necesariamente en la construcción, organización simbólica y afirmación cultural de una amplia gama de grupos juveniles y sus particulares formas de expresión, reflejadas en la música, la vestimenta, lenguaje; entre otras características (Mariani, S/F).

Cabe mencionar que la interacción con el mercado y la consagración cultural de modos de expresión de las juventudes no fue ni es determinante. Se debe considerar, además, que hay distintos medios de adquisición de bienes que no se relacionan con el mercado formal, porque hay maneras alternativas de producción y consumo, no mercantilizadas: economías informales, trueque, adaptación simbólica de bienes en contextos específicos, por ejemplo.

De acuerdo a lo anterior, podemos considerar el supuesto de que hay dos espacios de consumo en los jóvenes: primero, un consumo que se desarrolla en un mercado formal u oficial, regulado por el or- 
den social y las instituciones y determinado por la cultura hegemónica; y segundo, un consumo relacionado con el ámbito subcultural de los jóvenes. Por consiguiente, existe una multiplicidad de consumos independientes e interrelacionados, que van dando consistencia a perspectivas de vida, identidades y formas de expresión específicas emanadas de la interacción constante entre estos dos espacios sociales.

Por lo tanto, el cuerpo conceptual que expondremos a continuación debe suponer la relación explícita entre un consumo en el mercado formal y un consumo subcultural como espacios sociales que influyen activamente en la conformación de identidad(es) y una cultura en ese ámbito en los jóvenes. La consideración anterior nos permitirá establecer un marco de análisis de base para comprender que los procesos de consumo en la juventud son una dinámica de integración social y grupal; vale decir, procesos que permiten la integración a la sociedad en su conjunto y a los grupos de referencia de los individuos.

\section{JUVENTUD Y COMPONENTES PSICOSOCIALES Y CULTURALES DEL CONSUMO}

Cuando a la «juventud» se la ha considerado en términos de una categoría social específica, se ha hecho referencia a determinado periodo de transición entre la dependencia familiar y la vida adulta, que conlleva una serie de cambios físicos, mentales y socioculturales. Con la finalidad de delimitar este período de transición, los estudios sobre ella se centraron en aspectos etarios y en el concepto de moratoria, considerada como la prolongación de la dependencia parental. En Chile, la investigación tradicional se llevó a cabo, inicialmente, en términos de su impacto demográfico, y posteriormente se consideraron variables sociodemográficas (Tijoux, 1995; Alpízar y Bernal, 2003; Paz, 2004; Sandoval, 2005).

En otro contexto, la vivencia de esta etapa en la época moderna ha permitido que se lleven a cabo formas diversificadas de agrupación y expresión juvenil, que toman consistencia cultural. Así, se consideran culturas juveniles a los grupos de jóvenes que asimilan ciertos elementos actitudinales, simbólicos, conductuales, que van dando forma a estilos de vida específicos e independientes, reconocibles en determinado tiempo y espacio. Hay, por consiguiente, aspectos compartidos que permiten articular las relaciones entre los sujetos, el grupo y la sociedad en su conjunto. Igualmente, podemos agregar que las culturas juveniles se estructuran en función de la relación presente 
entre la cultura hegemónica, las culturas parentales, las culturas generacionales y una correspondencia de elementos sociales de pertenencia, entre otros: género, etnia, nacionalidad, clase, sexualidad, ideología, territorio (Feixa, 1996; Mariani, S/F).

Dependiendo del elemento cultural, o mejor, subcultural de las juventudes, cobra vital relevancia la dinámica de expresión simbólica en la constitución del estilo a través de procesos de consumo. El estilo es considerado como la organización activa y dinámica de un conjunto de elementos materiales e inmateriales compartidos por los jóvenes, los cuales se constituyen en medios de identidad del grupo. De esta manera, el estilo se puede manifestar en términos espectaculares, o bien, mediante formas privativas o individuales (Feixa, 1996).

Podemos considerar que a través de la adquisición de mercancías se lleva a cabo una asimilación cognitiva de símbolos y significados atribuidos a los objetos de consumo. En esta integración, se abstraen las propiedades simbólicas del objeto hacia el sujeto, permitiendo la personalización de la identidad considerada como lógica de consistencia del mundo privado del individuo mediante procesos de consumo (Baudrillard, 1969).

Según ello, podemos considerar que se establece una correspondencia específica entre la oferta de bienes en el mercado mediante el marketing empresarial, que trata de condicionar el consumo de un grupo objetivo de consumidores, y el manejo simbólico que realizan los jóvenes de los bienes que adquieren a la hora de dar consistencia a sus modos de vida. De allí que se considere que hay una relación cada vez más estrecha entre la identidad de marca de las mercancías, el mensaje publicitario — que a la vez es un objeto de consumo-, las formas de agregación juvenil y las comunidades temáticas de consumo que toman consistencia en nuestras sociedades actuales (Mariani, S/F).

El objeto de consumo comunica una identidad específica que, al mismo tiempo, se vincula con un sentido de pertenencia. Así, se puede comprender que existe una red compleja de símbolos atribuidos a las mercancías, los cuales comunican ciertos valores e ideales sociales que permiten dar significado y consistencia a la interacción social. No obstante, la oferta de bienes de consumo trasciende las barreras sociales, llegando indiscriminadamente a todos los estratos sociales económicamente desiguales. Obviamente, hay diferencias adquisitivas que provocan frustración y conflictos sociales específicos (Sandoval, 2005). De allí que el mercado de consumo sea considerado como excluyente en sí mismo. 
Por consiguiente, resulta de suma importancia delimitar el producto de las relaciones que se establecen entre los patrones de consumo en los jóvenes y las formas mercantilizadas de identidad que oferta el mercado.

Siguiendo el raciocinio anterior, podemos considerar el consumo en los y las jóvenes un mecanismo de mediación en la construcción y expresión de sus identidades y también un mecanismo de integración tanto al grupo de pares como a la sociedad en su conjunto. Ambos procesos de integración están directamente interrelacionados.

Ahora pasaremos a considerar un conjunto de elementos psicosocioculturales presentes en las sociedades que se rigen por un capitalismo de consumo, cuyos elementos conceptuales permitirán abordar analíticamente la forma que adopta el consumo en los jóvenes.

\section{Elemento material en la construcción de la identidad}

La construcción de la identidad es un proceso dinámico mediante el cual la persona integra el medio social como un espacio de referencia por el cual juzga el sí mismo, ya que pasa a formar parte integral del individuo, a la vez que un medio externo de referencia. En este sentido, la identidad se expresa según el modo en que los otros reconocen al sujeto y el autorreconocimiento, a su vez, dependerá de ese reconocimiento externo.

De acuerdo a Larraín (2001), la construcción de la identidad de una persona se estructura de acuerdo a tres elementos constitutivos: categorías socialmente compartidas, los otros significantes y un elemento material.

Las categorías sociales permiten a la persona identificar y hacer suyos ciertos elementos culturalmente compartidos que definen a una categoría social en específico. De tal manera que mediante un mecanismo de lealtad compartida hacia el grupo — según elementos y características reconocibles del mismo - la persona asume determinada cultura, lo que le permite arrogarse cierta identidad. De ahí deviene la idea de identidades culturales: etnia, religión, nacionalidad, etcétera.

Los otros significantes se consideran en una doble acepción. La construcción de la identidad de la persona se efectúa mediante la proyección de la opinión que otros tienen sobre el sí mismo del sujeto, en la medida en que le son significativamente importantes, a la hora de construir y reafirmar su identidad. Sólo cuentan aquellos grupos o personas con importante valor de referencia, quienes van estructuran- 
do el otro generalizado, que no es más que el sí mismo completo del sujeto (Larraín, 2001:28-29). No obstante, la construcción de la identidad, al momento de considerar a otros, conlleva al mismo tiempo al grupo de referencia a través del cual la persona traza diferencias relevantes en cuanto a modos de vida, valores, entre otras características. Por consiguiente, se delinean contrastes trascendentales, en cuanto representan a un grupo de oposición por el cual el sí mismo del sujeto reafirma su identidad.

El elemento material - al cual nos vamos a enfocar por su directa relación con los procesos de consumo - hace mención al hecho de que hay ciertos componentes materiales que posibilitan el autorreconocimiento por parte de la persona. Acceder a bienes materiales, sea por producción, sea por adquisición, permite a los sujetos exteriorizar, proyectar una extensión de sí mismos y de sus cualidades individuales atribuidas por los bienes que poseen. Consecuentemente, la construcción de la identidad se relaciona, en el caso del consumo de mercancías, con un acto culturalmente significativo que permite al sujeto la identificación y reconocimiento con un grupo social en específico.

En el consumo, los sujetos se adjudican las propiedades simbólicas de los objetos. Según ello, se favorece un proceso de comunicación que toma consistencia en la relación y diferenciación simbólica inherente al conjunto de bienes y servicios de consumo existentes en el mercado, ya que los mismos objetos de consumo son constituidos como elementos de códigos que permiten a los sujetos realizar cálculos para la formación, consistencia y diferenciación de su identidad (Baudrillard, 1969). La adquisición de mercancías se expone frente a otros, comunicando ciertos ideales o valores atribuidos a las personas mediante la extensión simbólica de los bienes que posee. Por ende, podemos considerar que los bienes establecen un estatus subjetivo e intersubjetivo en la interacción.

Las identidades son proyectos llevados a cabo por las personas, en donde su realización se considera una tarea constante y necesaria para el reconocimiento social. Esta identidad consiste, necesariamente, en una búsqueda, cuyos resultados se exponen gracias a marcas de pertenencia que el mercado ofrece.

No obstante, esa misma identidad, al igual que los medios simbólicos que ayudaron a conformarla, depende de las fluctuaciones del mercado -asimismo, su valoración-, lo que propicia que al menor cambio en la oferta se vuelva obsoleta, implicando, necesariamente, que sea temporal. Por ello, resulta más adecuado hablar de identida- 
des, pues las identidades rígidas, no proclives al cambio, son devaluadas socialmente, con lo que se genera un estado de emergencia y ambigüedad; una confusión permanente en las identidades de los sujetos, que pasan a ser privatizadas por la oferta que existe en el mercado (Bauman, 2005; 2007).

Por otro lado, según Lipovetsky (1990; 2002), se puede considerar que las personas están abiertas a los cambios y a la aceptación de lo nuevo, porque modifican sus modos de vida sin resistencias. Eso lleva a considerar aquellas personalidades e identidades, o a los modos de vida modernos, como esencialmente cinéticos: a través del consumo se acepta, se adquiere y se lleva a cabo la idea de cambio social y la transformación personal como un valor en sí mismo.

La racionalización del sujeto frente a la construcción de su(s) identidad(es) se orienta hacia el reciclaje: somete su vida e identidad al mantenimiento y al test. Vale decir, la persona se evalúa según los parámetros de los cambios experimentados en el mercado y en la moda. Así, existe una constante fragmentación del Yo que obedece a una lógica serial de yuxtaposiciones. Por consiguiente, la persona se considera una estructura abierta y dinámica ajena a los lazos de dependencia social, lo que provoca seres flotantes, cinéticos, que encuentran anclaje en proyectos de vida mercantilizados y temporales.

Lo anterior nos permite comprender por qué ciertas modas urbanas que aparecen en el espacio social son tan efímeras. Los consumidores ideales del mercado siguen la lógica de nacimientos y muertes de estilos de vida esencialmente comerciales. Estas identidades promocionadas por el mercado se manifiestan en ofertas dirigidas primordialmente a generar una demanda específica en un tiempo determinado. De esta forma, las personas que se rigen por la última moda saben valorar su entorno social — su grupo de pares y de referenciasegún la adscripción que realicen hacia las propiedades simbólicas de los últimos bienes de consumo que aparecen en el mercado. Esta identidad es un proyecto condenado a las constantes sucesiones.

Por lo tanto, podemos considerar un proceso de conversión y reconversión llevado a cabo mediante la constante yuxtaposición de identidades, lo cual forma personas cinéticas que adoptan estas variaciones como un imperativo categórico.

De igual manera, esta constante yuxtaposición ha permitido la extensión de la lógica del mercado a la esfera privada de los sujetos, lo que permite que las personas integren procesos de obsolescencia acelerada presentes en las variaciones de la oferta. La obsolescencia alude 
a la constante movilidad y proliferación de alternativas de consumo, su adquisición y la eliminación sistemática de los bienes. De acuerdo a ello, los cambios constantes y metódicos en las mercancías han llevado a una desvalorización del ideal de permanencia, de lo estable, en donde la norma es la valoración hacia lo nuevo en sí mismo (Lipovetsky, 1990; 2002).

En las sociedades de consumo emerge, en consecuencia, una moral dirigida hacia la transición obligada de los elementos-símbolos que favorecen la interacción social. De acuerdo a ello, el refugio, amparo, no estará reflejado en la adscripción a modelos tradicionales de vida reflejados por la estabilidad de sus elementos, sino, más bien, en el permanente reciclaje de sus personalidades supeditadas al constante nacimiento y muerte de bienes de consumo adquiridos en el mercado (Baudrillard, 1969). Así, la estabilidad en la vida moderna se hallará relacionada con la capacidad de vivir en lo sucesivo, a adaptarse de la mejor manera posible a las variaciones del mercado.

Se considera que los imperativos de la sociedad de consumo favorecen la fugacidad de los compromisos. Según esto, la relación establecida con los bienes adquiridos en el mercado debe obedecer a una satisfacción inmediata de necesidades en el mismo proceso de compra, donde no es necesario el valor tradicional de estabilidad, pues se lo considera disfuncional.

Existe, por tanto, la persistente necesidad de aumentar el volumen de las posesiones, de experimentar nuevos deseos, experiencias, y el reemplazo permanente de los bienes. Por ello, toda la simbología integrada al objeto de consumo trae consigo fecha de vencimiento, obsolescencia integrada y dirigida por la oferta cambiante. Así, la sociedad de consumo actúa hacia la insatisfacción constante de los consumidores, y esta insatisfacción se lleva a cabo mediante la devaluación de los bienes mediante publicidad, marketing, medios de comunicación, entre otros (Bauman, 2005, 2006, 2007, 2008).

\section{Mecanismos de integración-exclusion social a través del consumo}

La sociedad de consumo remite hacia - o pretende representar - un ideal de democracia que intenta ser vínculo con un ideal de progreso y promoción de posibilidades de acceso universal a los bienes de consumo. Podemos considerar que los mensajes que comunican los bienes alcanzan a todos los estratos sociales y son integrados constantemente 
en la vida cotidiana de las personas. Por eso, la capacidad de elección de los sujetos se presenta a priori.

Se supone que el acto de llevar a cabo una elección se encuentra disponible para todos mediante distintos mecanismos de adquisición - por capacidad real de consumo o créditos - lo que exige y obliga, al mismo tiempo, a entrar en el sistema cultural. De acuerdo a ello, la elección es una actividad específica que sentimos más como libertad que como obligación. De ahí que la elección remita hacia el orden político y económico de la sociedad, en cuanto la capacidad real de elección está distribuida desigualmente entre los estratos sociales (Baudrillard, 1969).

En cierto sentido, la persona posee, efectivamente, libertad dentro del entramado de oferta existente en el mercado. Pero el problema no lo constituye la elección en sí misma, sino el hecho de que debe, tiene y necesita llevar a cabo una elección o corre el riesgo latente de exclusión. De la misma forma, esta libertad de elección representa un mecanismo de estratificación social, por cuanto a mayor parámetro de opciones presentes en el mercado (donde la persona puede optar y ejercer sin restricciones la elección-apropiación de bienes), más alto será el lugar que ocupe en la escala social (Bauman, 2005, 2007, 2008).

Según la lógica de las ideas precedentes, debemos considerar que la elección de consumo dentro del mercado se presenta básicamente como una inversión que lleva a cabo el sujeto. En las sociedades con capitalismo de consumo, las personas elevan el valor de mercado ${ }^{1}$ de sí mismas mediante la adquisición de bienes. De allí que se considere

1 Elevar el valor de mercado de sí mismo a través del consumo hace referencia a la constitución de las personas como un objeto de consumo antes de que sujeto. El individuo es objeto de consumo que adquiere valor de venta de sí mismo a través del consumo de bienes, lo que le permite generar una oferta frente a sus otros significativos que demandan un «tipo» de sujeto particular. Asimismo, le posibilita integrarse socialmente. La noción de valor de venta de sí mismo presume la idea de considerar el valor de cambio semántico de los bienes (Ibáñez, 1994). En toda sociedad de consumo, las mercancías poseen un valor de uso, que hace referencia a la utilidad práctica; un valor de cambio económico, en cuanto a la retribución monetaria del bien, y un valor de cambio semántico, el cual alude a un conjunto de propiedades simbólicas que poseen los bienes y que se relaciona con lo que dicen o comunican sobre sus propietarios, y que son significativas en la interacción con otros. 
el objeto de consumo como un medio de integración social. No obstante, cabe considerar que para ganarse el estatus de consumidor y ejercer los derechos relacionados, la persona debe ser, antes que todo, un producto u objeto de consumo capaz de generar una demanda específica. Actuar de modo diferente conlleva pena de exclusión, por el hecho de que el individuo no ha podido elevar el valor de venta de sí mismo (Bauman, 2005, 2007, 2008). De ahí también que se considere que la apropiación de los bienes presentes en la oferta está guiada más por intereses estéticos que éticos, porque los primeros conforman un medio de integración esencial en una cultura que fomenta el consumo.

Así, en las personas existe el miedo a ser inadecuadas socialmente entre sus pares y este sentimiento es capitalizado por el mercado, que otorga las marcas de pertenencia a los grupos.

Por último, cabe considerar que la exclusión es una condición sicológica y social. El bienestar y la felicidad se juzgan de acuerdo a parámetros socialmente valorados, donde la incapacidad e imposibilidad de las personas de alcanzar aquellos estándares de gratificación genera necesariamente angustia y sufrimiento (Bauman, 2008).

\section{El sujeto como objeto de consumo}

En las sociedades de consumo hay una ideología subyacente en el objeto de consumo que se fundamenta en la noción de funcionalidad. Todo en el sistema social tiende hacia ella, en una suerte de integración de elementos en un conjunto que busca la replicación del organismo en específico. De allí que se considere que el objeto de consumo está integrado en un orden que satisface las necesidades de los individuos. No obstante, esta función primaria del objeto destinado al consumo — servicio de satisfacción de necesidades - se posterga por una función secundaria que está relacionada más por la combinación, control y manipulación de signos. (Baudrillard, 1969).

Mediante el consumo, el sujeto reafirma y construye su identidad por la vía del estatus simbólico atribuido al objeto. Por eso, se considera que hay una demanda social de personalidades mediada por los bienes de consumo. Sin embargo, debemos recordar que estos se vuelven obsoletos material y, más aún, simbólicamente, a consecuencia de la creciente oferta del mercado.

En la dinámica del consumo se produce un permanente reciclaje, conocimiento y muerte de los objetos que lo sustentan. Al desarrollar un consumo constante y variado, el individuo integra y elimina conti- 
nuamente las propiedades simbólicas de los bienes, lo que favorece un proceso de permanente reciclaje de la personalidad mediada por la connotación de las mercancías. Por ello, se considera que es el sujeto quien se transforma en objeto de demanda social y económica, porque incorpora para sí mismo las variaciones en la oferta del mercado. Según esta dinámica, se pueden considerar, además, que las diferencias estarán dadas por la población objetivo que se procura a través de la oferta, en donde la capacidad de elección será estructurada de antemano por los mismos productos que se ofrecen (Baudrillard, 1969).

De acuerdo a las ideas precedentes, las personas que integran el conjunto social pasan a ser consideradas como bienes de consumo, objetos. El individuo deberá internalizar en sí mismo la idea de un producto que se oferta de la mejor forma posible a fin de ganar el aprecio y la estima social. Según la gama de ofertas existente en el mercado, que prácticamente demanda un tipo de sujeto social, las personas deberán elevar su valor de mercado a través de un continuo consumo de bienes que eleven la atención de potenciales compradores; asimismo, el proceso delimita la pertenencia al grupo de referencia de los sujetos. De allí que se considere que hay un imperativo en las sociedades de consumo: nadie es consumidor — sujeto- , sin antes convertirse en producto —objeto—; la persona deberá integrar para sí misma la funcionalidad simbólica de los bienes y su constante transformación (Bauman, 2005, 2007, 2008).

Una persona eleva su valor de mercado a través del consumo de distintos bienes, pero deberá poseer una actitud permisiva al cambio e integrar un complejo número de símbolos variables atribuidos a los objetos. Esto le permitirá, a su vez, generar una oferta de sí misma. De ello se deriva que el objeto de consumo es constantemente superado por otros promovidos por el mercado, los cuales poseen nuevos valores impregnados que se transfieren a quienes los posean. Por eso que los consumidores ideales, para transformarse en objetos de demanda constante, reciclan su imagen a semejanza de las atribuciones relacionadas con los bienes, lo que les permite integrarse a un espacio social en constante transformación. Por lo tanto, la adscripción al modelo de mercado en la construcción de las identidades es un prerrequisito funcional que media relaciones sociales de todo tipo (Bauman, 2005, 2007, 2008).

Por consiguiente, la persona, concebida como producto que busca constantemente elevar su valor de mercado frente a otros, sigue con atención las variaciones del mercado de bienes de consumo de modo 
de aumentar su atractivo personal. Asimismo, esta conversión del sujeto en objeto hay que considerarla dentro de un complejo de relaciones con los componentes de la cultura del consumo que se integran en la construcción de las identidades y, a la vez, suponer que la dinámica del mercado se involucra en las relaciones interhumanas.

\section{Bricolage y homología}

Desde la semiótica, se pueden considerar dos elementos de análisis (Feixa, 1996) que permiten comprender la base cultural de la juventud que se expresa en la conformación del estilo (sistema organizado de elementos materiales e inmateriales que otorgan identidad de grupo): el bricolage y la homología.

El primero se define como la organización de objetos y símbolos presentes en un contexto cultural, que son reinterpretados según parámetros culturales propios del grupo. Aquella conversión se manifiesta en alteración de objetos, inversión y exageración de significados y sistemas de arreglo según un lenguaje propio del grupo.

La homología se entiende como una asociación de objetos, estilo e identidad que define a un grupo específico. O sea, se produce una agrupación de elementos que diferencian y estructuran un sistema de significados y acciones compartidos por los integrantes de ese grupo, lo que genera su identificación con determinados accesorios y ornamentos que pueden ser equivalentes a sus perspectivas asociativas.

\section{Posicionamiento y diferenciación}

De acuerdo a Mariani (S/F), en el estudio cultural de la juventud los patrones de consumo de sus integrantes permiten que se consideren parámetros de análisis propios de las empresas. El autor considera que existe una relación cada vez más estrecha entre las formas de agrupación de las juventudes y las actividades de marketing, que mediante estudios de mercado, publicidad y análisis cuantitativos y cualitativos delimitan la oferta hacia un segmento específico de consumidores. Según lo anterior, las formas de agrupación adolescente representan para las empresas nichos de consumidores, $\mathrm{y}$, al mismo tiempo, puntos de referencia a la hora de acometer una producción creativa.

Los estudios de mercado han desarrollado métodos y técnicas de investigación que procuran orientar la oferta y condicionar el consumo. Además, tratan de delimitar la oferta hacia grupos de consumido- 
res de referencia — segmentos-, como también de canalizar la demanda de consumidores potenciales. De acuerdo a ello, las empresas tratan de sintetizar los estilos de vida, valores y representaciones en determinadas marcas — productos- que los expresen. Esto es, construyen una identidad de marca — brand identiy-.

Por lo tanto, dos conceptos propios del marketing son considerados en la disposición cultural de la juventud en sus procesos de consumo: posicionamiento y diferenciación, que toman consistencia en los procesos de comercialización que intentan establecer una determinada identidad de marca.

Dentro de este contexto, posicionamiento y diferenciación están directamente relacionados. Al posicionar determinada marca — productose toma en cuenta su espacio de referencia y el de su competencia. Por eso se dice que el posicionamiento delimita las características del grupo de consumidores de referencia y define las propiedades del producto dentro de la amplia gama de oferta existente en el mercado, lo que posibilita su conexión con los compradores potenciales. Por consiguiente, se produce, al mismo tiempo, la diferenciación de la marca y la afirmación de su identidad en aquella diferencia.

En síntesis, se puede considerar un constante posicionamiento de bienes de consumo vinculados con determinadas formas de agrupación cultural de la juventud, merced a que las propiedades simbólicas de los objetos expresan características propias del grupo. Al mismo tiempo, estas prácticas permiten un proceso posicionamiento en los jóvenes, porque se establece una identidad grupal e individual mediante una diferenciación con otros grupos de referencia y/o de oposición.

\section{Sobre la publicidad}

Hay que considerar la publicidad, principalmente, como discurso y mensaje del mercado sobre los bienes de consumo. O sea, una connotación misma que integra una doble acepción: discurso y objeto de consumo a la vez. Su función principal, objetiva, es — o debería serla de comunicar las propiedades y características de los bienes. No obstante, con las propiedades simbólicas que comunica, su función secundaria — que remite a valores estéticos- califica y jerarquiza las relaciones sociales a través de la formalización de un sistema de significados accesibles a todos los grupos humanos, con lo cual permite que se establezcan puntos de referencia para evaluar el estatus social mediante la adscripción y adquisición de bienes (Baudrillard, 1969). 
La publicidad clasifica y, al mismo tiempo, ordena a los consumidores. Según ello, orienta una equivalencia y orden en los procesos de consumo (Ibáñez, 1994).

La equivalencia clasifica y delimita a los consumidores según las marcas/productos que comparten. Se da una afiliación según preferencias, que definen las fronteras del grupo. En el caso del sentido de orden, el consumo define una clase de filiación de los consumidores, los liga, los categoriza y los jerarquiza. La equivalencia separa, y el orden, agrupa.

Según lo precedente, se puede considerar la ficción de que los productos resultan accesibles para todos. Los bienes ya están dirigidos a consumidores específicos, lo cual mide las distancias en la escala social. Por consiguiente, se considera como paradójica la función de la publicidad: funciona, de manera oculta, de acuerdo a la clasificación, ordenamiento y mediación de los bienes. De acuerdo a ello, posee un mecanismo de coacción moralizante, pues produce la necesidad de consumir; y hay una función sugestiva que remite a la estética, produce el deseo de consumir (Ibáñez, 1994).

Por otro lado, se debe considerar que con los mensajes transmitidos por la publicidad se persigue una persuasión constante, que consigna la idea de un consumo esencialmente dirigido. Pero, por la gran cantidad de avisos publicitarios circulando en el espacio social, hay una reacción de saturación. De allí la idea de que el mensaje persuade y disuade al mismo tiempo. Sin embargo, la publicidad debe su eficacia a una lógica de fábula y adhesión: no se cree en los mensajes, pero las acciones son llevadas a cabo como si se creyesen en ellos. De ahí que se dirige una idea de protección y gratificación de los deseos mediante los bienes de consumo; el mensaje comunica un espacio donde los deseos, necesidades y gratificaciones se obtendrán mediante el consumo (Baudrillard, 1969).

Para concluir, podemos observar que la publicidad representa el mecanismo fundamental por el cual se comunican los valores socialmente relevantes que les son adjudicados a los bienes de consumo. Como consecuencia, establece parámetros simbólicos mediante los cuales las personas guían la construcción de su identidad. Asimismo, el mensaje consumido remite a un mundo idealizado, pero posible de alcanzar si se adoptan los medios que la publicidad pregona, y promociona constantemente sensaciones nuevas, lo que permite devaluar en forma sistemática la vida común que llevan las personas, porque las enfrenta a la opulencia y fascinación por bienes y artículos de consumo de última generación. 


\section{CONSIDERACIONES FINALES}

Podríamos decir que la cultura del consumo en Chile ya se ha instaurado en la idiosincrasia nacional, pues cada vez más hay una adscripción al consumo de bienes como medio de identificación social, autorrealización personal y como una estética de la vida, donde la relevancia de la simbología del objeto se ha vuelto un valor en sí mismo. Como menciona el Programa de las Naciones Unidas para el Desarrollo 2002, en nuestro país el consumo ha pasado a adquirir un «significado similar al que tenía antes el trabajo. Sería la cristalización física de la identidad individual, al tiempo que un nuevo anclaje material al vínculo social» (PNUD, 2002:98).

En este contexto de la sociedad chilena, el consumo se presenta de suma importancia en las formas de vida y desenvolvimiento social de la juventud. Podemos considerar la variación del endeudamiento en los jóvenes entre la V y VI Encuesta Nacional de la Juventud llevada a cabo por el Instituto Nacional de la Juventud (INJUV) en los años 2006 y 2009, respectivamente. De acuerdo a ello, para el estudio del año 2006 éste alcanzaba 33,18\% del total de la muestra, cifra que aumentó en 17 puntos porcentuales alcanzando $50,6 \%$ de endeudados para el año 2009 (INJUV, 2007, 2009). Este porcentaje de endeudamiento en los jóvenes, para el caso de la VI encuesta nacional, se asocia directamente con el consumo, distribuido entre casas comerciales - 57,3\%-, tarjetas de crédito - 33,7\% - y créditos de consumo - 20,7\%(INJUV, 2009). Si bien las cifras anteriores son referenciales, presentan un espacio objetivo de participación de los jóvenes.

Debemos considerar, además, que el análisis de las formas y/o consecuencias prácticas que adopta el consumo se halla aún en ciernes, tanto a nivel de la sociedad en su conjunto como de la juventud en particular. Por un extremo, se ha considerado que la masificación del consumo propicia una forma de ciudadanía mercantilizada, que responde a una constante despolitización y disciplinamiento de la sociedad (Moulian, 1997; Larraín, 2001). No obstante, estas consideraciones distan en la actualidad de contrastación empírica, lo que no implica necesariamente que sean menos relevantes.

De acuerdo al conocimiento sobre el consumo en los jóvenes y las formas que adopta, desarrollamos un marco de análisis que permita ser una base para una problematización ulterior de los procesos de consumo en la juventud, o bien, que oriente una comprensión inicial. 
Mencionamos que el consumo en la juventud se presenta principalmente como un proceso de construcción de las identidades y como integración social, al mismo tiempo. Dentro de este proceso, los jóvenes no llevan a cabo un consumo particular con el que puedan estructurar su identidad e integrarse socialmente, ni en un mercado en específico. Hay una dinámica constante de interacción de consumos que se desarrollan, básicamente, en dos espacios significativos. Como apuntábamos en los apartados iniciales de este ensayo, nos encontramos ante un consumo que se desarrolla en el mercado oficial y otro que se relaciona con el ámbito subcultural de la juventud, esto es, dentro de una economía informal.

Siguiendo lo anterior, el desarrollo del cuerpo de elementos teóricos los consideramos como interrelacionados, y toman consistencia en aquellos espacios donde se desenvuelve el consumo en los jóvenes. De allí la necesidad de tomar en cuenta los elementos estructurales de las sociedades de consumo y los componentes culturales de la juventud.

Según lo postulado, a modo de síntesis relacional, podemos mencionar que los procesos de conformación de la identidad dependerán de cómo los otros procesos significativos del sujeto reconozcan al individuo. Y el autorreconocimiento obedecerá a ese reconocimiento externo: la persona integra el medio social como el espacio de referencia por el cual se juzga a sí mismo. Para lograr aquel reconocimiento externo - permite a su vez elevar la autoestima—, el sujeto puede, a través del consumo, llevar a cabo una personalización de su identidad, pues el objeto, dotado de connotaciones sociales específicas, comunica una identidad que se relaciona, al mismo tiempo, con un sentido de pertenencia. Por lo tanto, las mercancías permiten una construcción y expresión de la identidad y, asimismo, son un mecanismo de integración al grupo de pares y a la sociedad en su conjunto.

Por la lógica variación de la oferta de bienes en el mercado, se considera que la construcción de la identidad es una tarea constante. La identidad, mediada por los bienes, necesita integrar la idea de cambio social y transformación personal con el carácter de prerrequisito funcional para desenvolverse socialmente en el mercado. Y tales características permiten calificar los modos de vida de las personas como esencialmente cinéticos, porque el cambio y la transformación personal son considerados valores en sí mismos. De acuerdo a ello, hay una constante fragmentación del Yo que obedece a un permanente proceso de conversión y reconversión de las personalidades por la necesidad de vivir en lo sucesivo. Así, se presentan procesos de obsolescencia de 
las identidades, que permite reconocer la extensión de la lógica del mercado a las relaciones sociales y al espacio privado de la vida.

Según lo razonado, el adoptar este cambio constante en la personalidad permitirá al individuo integrarse socialmente a un espacio de sucesivas transformaciones. Esta idea de variación continua permite interpretar la conformación del sujeto como objeto de consumo.

El medio social valora significativamente la adopción del modelo cultural. Y este aprecio social estará en directa relación con el reciclaje de la personalidad. O sea, el sujeto demanda, pero al mismo tiempo se ofrece según parámetros identitarios que determinan la pertenencia a un grupo social específico. En relación a ello, la persona aumenta el valor de mercado de sí misma con el consumo. Así, los bienes agrupan ciertos significados y valoraciones que asume el individuo, lo que le permite expresar un determinado estatus social.

Siguiendo la línea argumentativa precedente, las elecciones de consumo llevadas a cabo en el mercado se presentaran principalmente como una inversión, una garantía de integración social. La elección remite a la idea de adaptarse a los cambios que acontecen en el mercado. Con ello, se puede considerar que hay un estado de indeterminación social del sujeto que se remedia mediante el consumo. La persona lleva a cabo elecciones para solventar una exclusión y desvalorización social latente y que responden a una necesidad de integración social que se estructura como norma social.

La dinámica anterior de integración social y construcción de la identidad se puede vincular con la sociedad en su conjunto, pero, al mismo tiempo, con las formas de agregación juvenil. Asimismo, se puede suponer tanto con respecto a la expresión del grupo, como a la expresión privativa e individual de las personas.

Los grupos de jóvenes comparten ciertos elementos identitarios que articulan la relación entre sus miembros y con la sociedad en su conjunto. Vale decir, los procesos de consumo de mercancías permiten al individuo identificarse con cierto grupo de referencia, y al grupo expresar su identidad específica frente a otros conglomerados y frente a la sociedad.

Estas formas de expresión, tanto grupales como individuales, toman consistencia a través del estilo, el cual se estructura en procesos de bricolage y homología - no obstante, no son los únicos que permiten la disposición del estilo-, que están directamente relacionados con procedimientos de consumo. Así, el primero manipula y reinterpreta los significados de los objetos para otorgarles otros nuevos en un 
contexto determinado; y el segundo permite la asociación de un grupo con elementos identitarios específicos.

Asimismo, en la determinación identitaria a través de un consumo expresivo hay mecanismos de posicionamiento que permiten establecer un espacio referencial por el cual se delimitan las características propias del grupo y del sujeto, de manera que haya una diferenciación que permita afirmar una identidad específica.

Por último, cabe considerar la publicidad como discurso y mensaje del mercado con los cuales se devalúan socialmente los bienes de consumo. La publicidad es discurso y objeto de consumo que clasifica y jerarquiza las relaciones sociales con los bienes. Así, se presenta como un punto de referencia que evalúa el estatus social de las personas expuestas a su mensaje. Por consiguiente, se considera moralizante, pero al mismo tiempo sugestiva.

En definitiva, el marco analítico presentado permite contextualizar y considerar las relaciones de consumo que se establecen entre el individuo en particular y el nivel grupal, en relación con los parámetros culturales de una sociedad que adopta un capitalismo de consumo.

VALPARAÍSO (CHILE), MAYO 2010

RECIBIDO: MAYO 2010

ACEPTADO: JUNIO 2010

\section{REFERENCIAS BIBLIOGRÁFICAS}

ALPÍZAR, L. y M. BERNAL (2003): «La construcción social de las juventudes». Última Década №19. Viña del Mar: Ediciones CIDPA.

BAUDRILLARD, G. (1969): El sistema de los objetos. México: Siglo XXI.

BAUMAN, Z. (2008): La globalización. Consecuencias humanas. Buenos Aires: FCE.

(2007): Vida de consumo. Buenos Aires: FCE.

(2006): Modernidad líquida. Buenos Aires: FCE.

(2005). Trabajo, consumismo y nuevos pobres. Barcelona: Gedisa.

FEIXA, C. (1996): «De las culturas juveniles al estilo». Nueva Antropología, Revista de Ciencias Sociales №50.

IBÁÑEZ, J. (1994): Por una sociología de la vida cotidiana. Madrid: Siglo XXI. 
INJUV (2009): VI Encuesta nacional de la juventud. Principales resultados. Santiago: INJUV. (2007): V Encuesta nacional de la juventud. INJUV (2007).

LARRAÍN, J. (2001): Identidad chilena. Santiago: LOM.

LiPOVETSKY, G. (2002): La era del vacío. Barcelona: Anagrama.

- (1990): El imperio de lo efímero. La moda y su destino en las sociedades modernas. Barcelona: Anagrama.

MARIANI, NiCOLA (S/F): «Descifrar los jóvenes. Origen, evolución y límites del paradigma subcultural juvenil». Disponible en: http://red.enfocarte.com.

MARINAS, J. (2008): «Simmel y la cultura del consumo». Revista Española de Investigación Social (REIS) No89.

Moulian, T. (1997): Chile actual. Anatomía de un mito. Santiago: LOM.

PAZ, S. (2004): «Los jóvenes y la redefinición local de consumo». Última Década No21. Viña del Mar: Ediciones CIDPA.

PNUD (2002): Informe de desarrollo humano. Desarrollo humano en Chile. Nosotros los chilenos. Un desafío cultural. Santiago: PNUD.

SANDOVAL, M. (2005): «Jóvenes y exclusión (una difícil y compleja relación)». Santiago: Investigaciones CEJU, Centro de Estudios en Juventud UCSH.

TiJouX, M. (1995): «Jóvenes pobres en Chile: nadando en la modernidad y la exclusión». Última Década №3. Viña del Mar: Ediciones CIDPA. 